\title{
Ureteric Stone
}

National Cancer Institute

\section{Source}

National Cancer Institute. Ureteric Stone. NCI Thesaurus. Code C26902.

A concretion in the ureter. 